\title{
An Optimal Double Inequality among the One-Parameter, Arithmetic and Geometric Means
}

\author{
Hongya Gao, Shuangli Li, Yanjie Zhang, Hong Tian \\ College of Mathematics and Computer Science, Hebei University, Baoding, China \\ Email: ghy@hbu.cn, 563211828@qq.com,347764565@qq.com,602580999@qq.com
}

Received October 12, 2013; revised November 12, 2013; accepted November 17, 2013

Copyright (C) 2013 Hongya Gao et al. This is an open access article distributed under the Creative Commons Attribution License, which permits unrestricted use, distribution, and reproduction in any medium, provided the original work is properly cited.

\section{ABSTRACT}

In the present paper, we answer the question: for $0<\alpha<1$ fixed, what are the greatest value $p(\alpha)$ and the least value $q(\alpha)$ such that the double inequality $J_{p}(a, b)<\alpha A(a, b)+(1-\alpha) G(a, b)<J_{q}(a, b)$ holds for all $a, b>0$ with $a \neq b$ ? where for $p \in R$, the one-parameter mean $J_{p}(a, b)$, arithmetic mean $A(a, b)$ and geometric mean $G(a, b)$ of two positive real numbers $a$ and $b$ are defined by $J_{p}(a, b)= \begin{cases}a, & a \neq b, \\ \frac{p\left(a^{p+1}-b^{p+1}\right)}{(p+1)\left(a^{p}-b^{p}\right)}, & a \neq b, p \neq-1,0, \\ \frac{a b(\log a-\log b)}{a-b}, & a \neq b, p=-1, \\ \frac{a-b}{\log a-\log b}, & a \neq b, p=0,\end{cases}$ $A(a, b)=\frac{a+b}{2}$ and $G(a, b)=\sqrt{a b}$, respectively.

Keywords: Optimal Double Inequality; One-Parameter Mean; Arithmetic Mean; Geometric Mean

\section{Introduction}

For $p \in R$, the one-parameter mean $J_{p}(a, b)$, arithmetic mean $A(a, b)$ and geometric mean $G(a, b)$ of two positive real numbers $a$ and $b$ are defined by

$$
J_{p}(a, b)= \begin{cases}a, & a \neq b, \\ \frac{p\left(a^{p+1}-b^{p+1}\right)}{(p+1)\left(a^{p}-b^{p}\right)}, & a \neq b, p \neq-1,0, \\ \frac{a b(\log a-\log b)}{a-b}, & a \neq b, p=-1, \\ \frac{a-b}{\log a-\log b}, & a \neq b, p=0,\end{cases}
$$

$A(a, b)=\frac{a+b}{2}$ and $G(a, b)=\sqrt{a b}$, respectively.
It is well-known that the one-parameter mean is continuous and strictly increasing with respect to $p \in R$ for fixed $a, b>0$ with $a \neq b$. Many means are special cases of the one-parameter mean, for example: $J_{1}(a, b)=A(a, b)$ is the arithmetic mean, $J_{1 / 2}(a, b)=H e(a, b)$ is the Heronian mean, $J_{-1 / 2}(a, b)=G(a, b)$ is the geometric mean, and $J_{-2}(a, b)=H(a, b)$ is the harmonic mean.

The one-parameter mean $J_{p}(a, b)$ and its inequalities have been studied intensively, see [1-6].

The purpose of this paper is to answer the question: for $0<\alpha<1$, what are the greatest value $p(\alpha)$ and the least value $q(\alpha)$ such that the double inequality

$J_{p}(a, b)<\alpha A(a, b)+(1-\alpha) G(a, b)<J_{q}(a, b)$ holds for all $a, b>0$ with $a \neq b$ ? 


\section{Main Result}

The main result of this paper is the following theorem.

Theorem 2.1. Let $0<\alpha<1$. Then for any $a, b>0$ with $a \neq b$, we have

1)

$$
\begin{aligned}
& J_{\frac{3 \alpha-1}{2}}(a, b)=\alpha A(a, b)+(1-\alpha) G(a, b)=J_{\frac{\alpha}{2-\alpha}}(a, b) \text { for } \\
& \alpha=\frac{2}{3},
\end{aligned}
$$

$J_{\frac{3 \alpha-1}{2}}(a, b)<\alpha A(a, b)+(1-\alpha) G(a, b)<J_{\frac{\alpha}{2-\alpha}}(a, b)$ for $\alpha \in\left(0, \frac{2}{3}\right)$,

3)

$J_{\frac{\alpha}{2-\alpha}}(a, b)<\alpha A(a, b)+(1-\alpha) G(a, b)<J_{\frac{3 \alpha-1}{2}}(a, b)$ for $\alpha \in\left(\frac{2}{3}, 1\right)$.

The numbers $\frac{3 \alpha-1}{2}$ and $\frac{\alpha}{2-\alpha}$ in 2) and 3) are optimal.

In order to prove Theorem 2.1, we need a preliminary lemma.

Lemma 2.1. For $t>1$, one has

$$
g(t)=\frac{t^{2}-1}{2 \log t}-\frac{t^{2}+4 t+1}{6}<0
$$

Proof. Simple calculations lead to

$$
\begin{gathered}
g(t)=\frac{t^{2}+4 t+1}{6 \log t} g_{1}(t), \\
g_{1}(t)=\frac{3\left(t^{2}-1\right)}{t^{2}+4 t+1}-\log t, \\
\lim _{t \rightarrow 1^{+}} g_{1}(t)=0, \\
g_{1}^{\prime}(t)=\frac{-t^{4}+4 t^{3}-6 t^{2}+4 t-1}{t\left(t^{2}+4 t+1\right)^{2}}=\frac{-(t-1)^{4}}{t\left(t^{2}+4 t+1\right)^{2}}<0
\end{gathered}
$$

(2) follows from (3)-(6).

Proof of Theorem 2.1. Without loss of generality we assume $a>b$ and take $t=\sqrt{a / b}>1$. We first consider the case $\alpha=\frac{2}{3} .1$ ) follows from

$$
J_{\frac{1}{2}}(t, 1)=H e(t, 1)=\frac{t+\sqrt{t}+1}{3}=\frac{2}{3} A(t, 1)+G(t, 1) .
$$

From now on we assume $\alpha \neq \frac{2}{3}$. Let

$$
\begin{aligned}
p \in\left\{\frac{3 \alpha-1}{2}, \frac{\alpha}{2-\alpha}\right\}, \text { then (1) leads to } \\
f(t)=\left[\alpha A\left(t^{2}, 1\right)+(1-\alpha) G\left(t^{2}, 1\right)\right]-J_{p}\left(t^{2}, 1\right) \\
=\frac{h(t)}{2(p+1)\left(t^{2 p}-1\right)},
\end{aligned}
$$

where

$$
\begin{aligned}
h(t)= & (\alpha p-2 p+\alpha) t^{2 p+2}+2(1-\alpha)(p+1) t^{2 p+1} \\
& +\alpha(p+1) t^{2 p}-\alpha(p+1) t^{2} \\
& -2(1-\alpha)(p+1) t-(\alpha p-2 p+\alpha) .
\end{aligned}
$$

Simple calculations lead to

$$
\lim _{t \rightarrow 1^{+}} h(t)=0,
$$

$$
\begin{aligned}
h^{\prime}(t)= & 2(p+1)(\alpha p-2 p+\alpha) t^{2 p+1} \\
& +2(2 p+1)(1-\alpha)(p+1) t^{2 p}+2 \alpha p(p+1) t^{2 p-1} \\
& -2 \alpha(p+1) t-2(1-\alpha)(p+1) \\
= & 2(p+1) h_{1}(t),
\end{aligned}
$$

where

$$
\begin{gathered}
h_{1}(t)=(\alpha p-2 p+\alpha) t^{2 p+1}+(1-\alpha)(2 p+1) t^{2 p} \\
+p \alpha t^{2 p-1}-\alpha t-(1-\alpha) \\
\lim _{t \rightarrow 1^{+}} h_{1}(t)=0 \\
h_{1}^{\prime}(t)=(2 p+1)(\alpha p-2 p+\alpha) t^{2 p} \\
+2 p(1-\alpha)(2 p+1) t^{2 p-1} \\
+p(2 p-1) \alpha t^{2 p-2}-\alpha \\
\quad \lim _{t \rightarrow 1^{+}} h_{1}^{\prime}(t)=0 \\
h_{1}^{\prime \prime}(t)=2 p(2 p+1)(\alpha p-2 p+\alpha) t^{2 p-1} \\
+2 p(2 p-1)(1-\alpha)(2 p+1)) t^{2 p-2} \\
+2(p-1) p(2 p-1) \alpha t^{2 p-3} \\
=2 p t^{2 p-3} h_{2}(t)
\end{gathered}
$$

where

$$
\begin{aligned}
& h_{2}(t)=(2 p+1)(\alpha p-2 p+\alpha) t^{2} t \\
&+(2 p-1)(2 p+1)(1-\alpha) \\
&+(p-1)(2 p-1) \alpha \\
& \lim _{t \rightarrow 1^{+}} h_{2}(t)=3 \alpha-2 p-1, \\
& h_{2}^{\prime}(t)=2(2 p+1)(\alpha p-2 p+\alpha) t \\
&+(2 p-1)(2 p+1)(1-\alpha) \\
&=(2 p+1) h_{3}(t),
\end{aligned}
$$


where

$$
\begin{gathered}
h_{3}(t)=2(\alpha p-2 p+\alpha) t+(2 p-1)(1-\alpha), \\
\lim _{t \rightarrow 1^{+}} h_{3}(t)=3 \alpha-2 p-1, \\
h_{3}^{\prime}(t)=2(\alpha p-2 p+\alpha) .
\end{gathered}
$$

We shall distinguish between two cases.

Case 1. $p=\frac{3 \alpha-1}{2}$. The left-hand side inequality of 2) for $\alpha=\frac{1}{3}$ follows from Lemma 2.1 because in this case $J_{0}\left(t^{2}, 1\right)-\left[\frac{1}{3} A\left(t^{2}, 1\right)+\frac{2}{3} G\left(t^{2}, 1\right)\right]=g(t)<0$

for all $t>1$. In the sequel we assume $\alpha \neq \frac{1}{3}$.

We clearly see from (16) that

$$
h_{3}^{\prime}(t)=(3 \alpha-2)(\alpha-1)\left\{\begin{array}{l}
<0, \alpha \in\left(0, \frac{2}{3}\right), \\
>0, \alpha \in\left(\frac{2}{3}, 1\right) .
\end{array}\right.
$$

Thus $h_{3}(t)$ is strictly decreasing for $\alpha \in\left(0, \frac{2}{3}\right)$ and strictly increasing for $\alpha \in\left(\frac{2}{3}, 1\right)$. (2.14) yields $h_{3}\left(1^{+}\right)=0$, then $h_{3}(t)<0 \quad$ for $\quad \alpha \in\left(0, \frac{2}{3}\right) \quad$ and $h_{3}(t)>0$ for $\alpha \in\left(\frac{2}{3}, 1\right)$. The same reasoning applies to $h_{2}^{\prime}(t)$ and $h_{2}(t)$ as well, and noticing (13) and (12), one has

$$
h_{2}(t)\left\{\begin{array}{l}
>0, \alpha \in\left(0, \frac{2}{3}\right), \\
<0, \alpha \in\left(\frac{2}{3}, 1\right) .
\end{array}\right.
$$

This result together with (11) implies

$$
h_{1}^{\prime \prime}(t)\left\{\begin{array}{l}
<0, \alpha \in\left(0, \frac{1}{3}\right) \cup\left(\frac{2}{3}, 1\right), \\
>0, \alpha \in\left(\frac{1}{3}, \frac{2}{3}\right) .
\end{array}\right.
$$

Thus $h_{1}^{\prime}(t)$ is strictly decreasing for $\alpha \in\left(0, \frac{1}{3}\right) \cup\left(\frac{2}{3}, 1\right)$ and strictly increasing for $\alpha \in\left(\frac{1}{3}, \frac{2}{3}\right)$. The same reasoning applies to $h_{1}^{\prime}(t), h_{1}(t)$ and $h(t)$ as well, and applying (8)-(10), we derive

$$
h(t)\left\{\begin{array}{l}
<0, \alpha \in\left(0, \frac{1}{3}\right) \cup\left(\frac{2}{3}, 1\right), \\
>0, \alpha \in\left(\frac{1}{3}, \frac{2}{3}\right) .
\end{array}\right.
$$

Since $t^{2 p}-1<0$ for $\alpha \in\left(0, \frac{1}{3}\right)$ and $t^{2 p}-1>0$ for $\alpha \in\left(\frac{1}{3}, 1\right)$, then we know from (7) that

$$
f(t)\left\{\begin{array}{l}
>0, \alpha \in\left(0, \frac{2}{3}\right) \\
<0, \alpha \in\left(\frac{2}{3}, 1\right) .
\end{array}\right.
$$

This implies the left-hand side of 2) and the right-hand side of 3).

Case 2. $p=\frac{\alpha}{2-\alpha}$. From (14) we know that

$$
h_{3}(t)=\frac{(3 \alpha-2)(1-\alpha)}{2-\alpha}\left\{\begin{array}{l}
<0, \alpha \in\left(0, \frac{2}{3}\right) \\
>0, \alpha \in\left(\frac{2}{3}, 1\right) .
\end{array}\right.
$$

From (13) we know that $h_{2}^{\prime}(t)<0$ for $\alpha \in\left(0, \frac{2}{3}\right)$ and $h_{2}^{\prime}(t)>0$ for $\alpha \in\left(\frac{2}{3}, 1\right)$. This implies $h_{2}(t)$ is strictly decreasing for $\alpha \in\left(0, \frac{2}{3}\right)$ and strictly increasing for $\alpha \in\left(\frac{2}{3}, 1\right)$. From (12) we know

$$
\lim _{t \rightarrow 1^{+}} h_{2}(t)=\frac{(3 \alpha-2)(1-\alpha)}{2-\alpha}\left\{\begin{array}{l}
<0, \alpha \in\left(0, \frac{2}{3}\right) \\
>0, \alpha \in\left(\frac{2}{3}, 1\right) .
\end{array}\right.
$$

Therefore

$$
h_{2}(t)\left\{\begin{array}{l}
<0, \alpha \in\left(0, \frac{2}{3}\right) \\
>0, \alpha \in\left(\frac{2}{3}, 1\right) .
\end{array}\right.
$$

(11) implies $h_{1}^{\prime \prime}(t)$ has the same property as $h_{2}(t)$, thus $h_{1}^{\prime}(t)$ is strictly decreasing for $\alpha \in\left(0, \frac{2}{3}\right)$ and strictly increasing for $\alpha \in\left(\frac{2}{3}, 1\right)$. The same reasoning applies to $h_{1}(t), h^{\prime}(t)$ and $h(t)$ as well, and notic- 
ing (9) and (8), one has

$$
h(t)\left\{\begin{array}{l}
<0, \alpha \in\left(0, \frac{2}{3}\right), \\
>0, \alpha \in\left(\frac{2}{3}, 1\right) .
\end{array}\right.
$$

which together with (7) implies

$$
f(t)\left\{\begin{array}{l}
<0, \alpha \in\left(0, \frac{2}{3}\right), \\
>0, \alpha \in\left(\frac{2}{3}, 1\right) .
\end{array}\right.
$$

This implies the right-hand side of 2) and the left-hand side of 3).

We are now in the position to prove the constants

$\frac{3 \alpha-1}{2}$ and $\frac{\alpha}{2-\alpha}$ are optimal.

For any $\quad \varepsilon$ (positive or negative, with $|\varepsilon|$ sufficiently small) we consider the case $p=\frac{3 \alpha-1}{2}+\varepsilon$. implies

$$
\lim _{t \rightarrow 1^{+}} h_{2}(t) \begin{cases}<0, & \varepsilon>0, \\ >0, & \varepsilon<0 .\end{cases}
$$

By the continuity of $h_{2}(t)$, there exists $\delta_{1}=\delta_{1}(\varepsilon)>0$ such that

$$
h_{2}(t)\left\{\begin{array}{l}
<0, \text { for } 1<t<1+\delta_{1} \text { and } \varepsilon>0, \\
>0, \text { for } 1<t<1+\delta_{1} \text { and } \varepsilon<0 .
\end{array}\right.
$$

By (11), $p h_{1}^{\prime \prime}(t)$ as the same property as $h_{2}(t)$. The same reasoning applies to $p h_{1}^{\prime}(t), p h_{1}(t), p h^{\prime}(t)$ and $p h(t)$ as well, and noticing (10)-(8), we know $p h(t)$ has the same property as $h_{2}(t)$. By (7) one has

$$
f(t) \begin{cases}<0, & \varepsilon>0 \\ >0, & \varepsilon<0 .\end{cases}
$$

This proves the optimality for $\frac{3 \alpha-1}{2}$.

To prove the optimality for $\frac{\alpha}{2-\alpha}$ in the right-hand side of 2) and the left-hand side of 3), we notice from

$$
\lim _{t \rightarrow \infty} \frac{\alpha A(t, 1)+(1-\alpha) G(t, 1)}{J_{p}(t, 1)}=\frac{\alpha(p+1)}{2 p} \begin{cases}<1, & p>\frac{\alpha}{2-\alpha}, \\ >1, & p<\frac{\alpha}{2-\alpha},\end{cases}
$$

that there exists $T \in(1, \infty)$ such that

$$
\alpha A(t, 1)+(1-\alpha) G(t, 1)<J_{P}(t, 1)
$$

for $p>\frac{\alpha}{2-\alpha}$ and $t \in(T,+\infty)$, and

$$
\alpha A(t, 1)+(1-\alpha) G(t, 1)>J_{P}(t, 1)
$$

for $t \in(T,+\infty)$. This ends the proof of Theorem 2.1.

\section{Acknowledgements}

This paper is supported by NSF of Hebei Province (A2011201011).

\section{REFERENCES}

[1] H. Y. Gao and W. J. Niu, "Sharp Inequalities Related to One-Parameter Mean and Gini Mean,” Journal of Mathematical Inequalities, Vol. 6, No. 4, 2012, pp. 545-555.

[2] W. S. Cheung and F. Qi, "Logarithmic Convexity of the One-Parameter Mean Values,” Taiwanese Journal of Mathematics, Vol. 11, No. 1, 2007, pp. 231-237.

[3] M. K. Wang, Y. F. Qiu and Y. M. Chu, “An Optimal Double Inequality among the One-Parameter, Arithmetic and Harmonic Means," Revue D'Analyse Numerique de Theorie de L'approximation, Vol. 39, No. 2, 2012, pp. 169-175.

[4] H. N. Hu, G. Y. Tu and Y. M. Chu, "Optimal Bouds for the Seiffert Mean in Terms of One-Parameter Means," Journal of Applied Mathematics, Vol. 2012, No. 1, 2012, Article ID: 917120.

[5] B. Y. Long and Y. M. Chu, "Optimal Inequalities for Generalized Logarithmic, Arithmetic and Geometric Mean,” Journal of Inequalities and Applications, Vol. 2010, No. 1, 2010, Article ID: 806825.

[6] N. G. Zheng, Z. H. Zhang and X. M. Zhang, Schur-Convexity of Two Types of One-Parameter Mean Values in Variables,” Journal of Inequalities and Applications, Vol. 2007, No. 1, 2007, Article ID: 78175. 